\title{
BMJ Open UK-based prospective cohort study to anglicise and validate the FACE-Q Skin Cancer Module in patients with facial skin cancer undergoing surgical reconstruction: the PROMISCR (Patient- Reported Outcome Measure in Skin Cancer Reconstruction) study
}

\author{
Thomas Dobbs, ${ }^{1,2}$ Hayley A Hutchings, ${ }^{3}$ Iain S Whitaker ${ }^{1,2}$
}

To cite: Dobbs T, Hutchings HA, Whitaker IS. UK-based prospective cohort study to anglicise and validate the FACE-Q Skin Cancer Module in patients with facial skin cancer undergoing surgical reconstruction: the PROMISCR (Patient-Reported Outcome Measure in Skin Cancer Reconstruction) study. BMJ Open 2017;7:e016182. doi:10.1136/ bmjopen-2017-016182

- Prepublication history for this paper is available online. To view these files, please visit the journal online (http://dx.doi.org/ 10.1136/bmjopen-2017-016182

Received 30 January 2017 Revised 17 August 2017

Accepted 22 August 2017

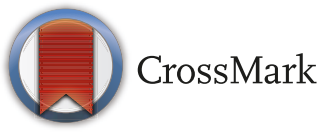

${ }^{1}$ Reconstructive Surgery \& Regenerative Medicine Research Group, Institute of Life Sciences, Swansea University Medical School, Swansea, UK

${ }^{2}$ Welsh Centre for Burns and Plastics, Morriston Hospital, Swansea, UK

${ }^{3}$ Department of Health Services Research, Institute of Life Sciences, Swansea University Medical School, Swansea, UK

Correspondence to

Thomas Dobbs;

tomdobbs@doctors.org.uk

\section{ABSTRACT}

Introduction Skin cancer is the most common malignancy worldwide, often occurring on the face, where the cosmetic outcome of treatment is paramount. $A$ number of skin cancer-specific patient-reported outcome measures (PROMs) exist, however none adequately consider the difference in type of reconstruction from a patient's point of view. It is the aim of this study to 'anglicise' (to UK English) a recently developed US PROM for facial skin cancer (the FACE-Q Skin Cancer Module) and to validate this UK version of the PROM. The validation will also involve an assessment of the items for relevance to facial reconstruction patients. This will either validate this new measure for the use in clinical care and research of various facial reconstructive options, or provide evidence that a more specific PROM is required. Methods and analysis This is a prospective validation study of the FACE-Q Skin Cancer Module in a UK facial skin cancer population with a specific focus on the difference between types of reconstruction. The face and content validity of the FACE- $Q$ questionnaire will initially be assessed by a review process involving patients, skin cancer specialists and methodologists. An assessment of whether questions are relevant and any missing questions will be made. Initial validation will then be carried out by recruiting a cohort of 100 study participants with skin cancer of the face pre-operatively. All eligible patients will be invited to complete the questionnaire preoperatively and postoperatively. Psychometric analysis will be performed to test validity, reliability and responsiveness to change. Subgroup analysis will be performed on patients undergoing different forms of reconstruction postexcision of their skin cancer.

Ethics and dissemination This study has been approved by the West Midlands, Edgbaston Research Ethics Committee (Ref 16/WM/0445). All personal data collected will be anonymised and patient-specific data will only be reported in terms of group demographics. Identifiable data collected will include the patient name and date of birth. Other collected personal data will include their
Strengths and limitations of this study

- This is a prospective study to anglicise and further validate a newly developed patient-reported outcome measures for facial skin cancer.

- A strength is that this study will follow international standards for psychometrically testing the new FACE-Q Skin Cancer Module.

- A limitation may be that the questions in the FACE-Q Skin Cancer Module are found to be not sensitive enough to show a difference between different reconstructive options for facial skin cancer.

diagnosis, treatment performed, method of reconstruction and complications. A unique identifier will be applied to each patient so that pretreatment and post-treatment questionnaire results can be compared. All data acquisition and storage will be in accordance with the Data Protection Act 1998. Following completion of the study, all records will be stored in the Abertawe Bro Morgannwg University (AMBU) Health Board archive facility. Only qualified personnel working on the project will have access to the data. The outputs from this work will be published as widely as possible in peer-review journals and it is our aim to make this open access.

\section{INTRODUCTION}

Skin cancer is the most common malignancy worldwide, ${ }^{1}$ with one in five Americans developing skin cancer in their lifetime. ${ }^{2}$ The non-melanoma skin cancers (NMSC) greatly outweigh the number of melanomas diagnosed. ${ }^{3}$ In 2013, over 72000 new NMSC diagnoses were made in the UK and approximately 3.3 million Americans are treated annually for NMSC. ${ }^{34}$ Of the NMSCs, around $80 \%$ of these lesions are located on the 
head and neck. ${ }^{5}$ Furthermore, it is predicted that the number of skin cancer diagnoses is going to continue to increase year-on-year, with a doubling in number in the next 30 years, along with a fall in the average age of first diagnosis. ${ }^{6}$

Treatment for skin cancer is wide ranging and while surgical excision with a margin of non-involved tissue is typically favoured in Europe and the USA, ${ }^{7}$ many other options such as electrodessication and curettage, topical or intralesional chemotherapy, ${ }^{8}$ radiotherapy ${ }^{9}$ and in some cases laser treatment exist. ${ }^{10}$ Five-year cure rates, especially for excisional treatment of NMSC, are generally good and therefore the challenge is often reconstructing the defect following surgical excision, especially on the head and neck where cosmesis is clearly important. ${ }^{11}$ Reconstructive options include direct closure, skin grafts, local tissue flaps and more rarely free flaps or allowing healing by secondary intention. Direct closure is commonly the most preferable option in terms of cosmesis and reduced postoperative complications (from a clinicians point of view), but in many cases it may not be possible.

In an era where shared decision-making between clinicians and patients is encouraged, ${ }^{12}$ it is important that the patient is involved in the discussion regarding the most appropriate reconstructive option. It is also important to recognise that health should be considered as 'a state of physical, mental and social well-being and not just the absence of disease or infirmity' and as such, the aesthetic and emotional outcome of various types of reconstruction is just as important as the removal of the skin malignancy. To date, very little work has focused on the patient perspective of the difference between these reconstructive options. While patient-reported outcome measures (PROMs) for skin cancer exist, these are often not specific to the reconstruction and do not explore patients' views between the different types of reconstruction.

PROMs are questionnaires where responses are collected directly from patients and are used to measure the perception and impact of a condition and its treatment on their quality of life or disease severity. They are considered by the UK Department of Health as currently the best method for quantifying a patients' clinical experience and are critical in maximising quality of care. Currently, only four surgical conditions have routine PROM data collected at a national level in the UK, although both generic and disease-specific PROMs are often employed at a local level and in clinical trials. ${ }^{13}$

A number of different PROMs have been applied to or designed for patients with skin cancer. A systematic review in 2013 demonstrated that there were two generic PROMs (SF-36 and Sickness Impact Profile) and nine skin cancer-specific PROMs (EORTC QLQ-C30, EORTC QLQ-M, FACT-M, FACT-BRM, SCI, FSCI, DLQI, Skindex and SCQOLIT). ${ }^{14}$ One of the limitations when these were evaluated was that for patients with NMSC, the scores on these measures are often comparable to population norms. It may be that there is no 'true' difference between those with NMSC and those without, however it is more likely that current PROMs are not capturing the important aspects of quality of life in this patient group. Recent work by Lee and colleagues have demonstrated the need for an improved and specific PROM for patients with skin cancer of the head and neck,${ }^{15}$ which they have developed in the form of the FACE-Q Skin Cancer Module. However, no PROM to date has been specifically evaluated for or designed to look at the difference between varying reconstructive options post-skin cancer resection. In order that we are able to appropriately council our patients on the different types of reconstruction and evaluate the differences from a patients' perspective, this situation needs to be addressed.

\section{AIMS AND OBJECTIVES}

The aim of this study is to anglicise and validate the FACE-Q Skin Cancer Module for use in a UK population of patients who are undergoing reconstruction for a facial skin cancer. Anglicisation is an important step in the adoption of this new PROM into UK practice as subtle differences in grammar and language style can influence the understanding and scoring of a PROM questionnaire. This is a well-documented process for using a PROM originally developed in another country or language. ${ }^{16}$ We will undertake preliminary psychometric analysis of the anglicised FACE-Q to test reliability, construct validity, test-retest reliability and responsiveness in three specific groups-those patients who have undergone reconstruction either with direct closure, skin grafting or a local skin flap. This is with the aim of determining if the FACE-Q Skin Cancer Module is able to detect a difference in patient outcomes between these three reconstructive options.

\section{METHODS AND ANALYSIS}

\section{Anglicisation of the FACE-Q Skin Cancer Module}

The FACE-Q Skin Cancer Module will be reviewed by a group of skin cancer specialists (including dermatologists, plastic surgeons and other members of the multidisciplinary team) and methodologists in order to determine the face and content validity of the instrument. Face validity refers to whether the questions appear to be assessing the desired qualities that is, are they on the surface measuring what they actually are, while content validity is a judgement assessment of whether the items in a scale encompass all relevant and important areas of the concept being measures in appropriate detail. To ensure that the questionnaire is understandable and readable to our population group a small cohort of patients will then be selected to review the questionnaire. This will be conducted in the form of 1-to-1 cognitive interviews, which will be arranged to coincide with normal clinical follow-up and will therefore not incur any additional costs to the patient. Each question will be scrutinised for language content appropriate to a UK-based cohort and if needed anglicised. Prior to this stage, a systematic 
review of the literature pertaining to both PROMs for facial skin cancer and PROMs for facial reconstruction will be conducted in order to identify any other questions which may be important in determining the difference between different reconstructive options for facial skin cancer. If additional questions are identified either through patient and clinician interviews or from other PROMs identified in systematic reviews, these will be included in the study for initial validation in our facial skin cancer cohort.

\section{Recruitment}

A cohort of at least 100 patients will be recruited prospectively from the plastic surgery department at Morriston Hospital, Swansea, UK over an 18-month period beginning in August 2018. Invitation to participate will be offered to all patients presenting with a skin cancer of the head and neck over the age of 18 years to the Plastic Surgery department. Eligibility criteria for inclusion will be based on:

\section{Inclusion criteria}

- Skin cancer (all types included) of the head and neck

- Over 18years of age

- Active treatment with wide local excision of the lesion.

\section{Exclusion criteria}

- Inability to consent to participation in study

- Known learning difficulties or dementia

- English language not of a standard to understand and complete the questionnaire

- Treatment of lesion with topical chemotherapy/laser or other methods that are not excisional

- Free tissue reconstruction.

Those patients who meet the inclusion criteria will be given an oral and written explanation of the study. If they wish to be involved, they will be asked to sign a consent form and this will be recorded in the patient notes. All participants will be free to withdraw their consent from the study at any point.

Each study participant will be asked to complete the FACE-Q skin cancer questionnaire either at the time of their clinic appointment or later at home. They will also complete the Facial Skin Cancer Index (FSCI) questionnaire $^{17}$ and the generic European Quality of Life Five Dimensions (EQ5D) questionnaire. ${ }^{18}$

\section{Sample size}

There are no general criteria for the required sample size when validating a PROM questionnaire ${ }^{19}$ although some believe that the number of respondents should be three times the number of items in the questionnaire. ${ }^{20}$ No matter what sample size is chosen it is however important that the sample is representative of the population group on which the instrument is intended to be used. We aim to recruit at least 100 patients so that we have $>30$ patients in each arm (eg, direct closure/skin graft/local flap).

\section{Psychometric analysis}

Psychometric analysis will be performed inline with the gold standards for instrument assessment as defined by the Scientific Advisory Committee of the Medical Outcomes Trust ${ }^{21}$ and methods outlined by Streiner and Norman. ${ }^{19}$ All data will be analysed using the Statistical Package for Social Sciences software licensed to Swansea University.

\section{Underlying dimensions and internal consistency}

Internal consistency is one measure of reliability. It measures the degree of correlation between different items in a scale and therefore questions that are asking similar things should correlate. We will assess the internal consistency of the anglicised FACE-Q by examining itemtotal correlations and Cronbach's $\alpha$. We will consider questions for rejection if their item-total correlations are $<0.2$ (hardly related) or $>0.8$ (highly related and therefore providing little additional information). ${ }^{19}$ Finally, we will examine Cronbach's $\alpha$ for the total questionnaire score and for any factor/sub-scale scores to ensure that they $>0.7 .^{19}$

We will then apply principal components analysis to the data. We will consider a factor important if its eigenvalue (the power to explain variation between patients) $>1$, and that it has face validity, meaning that it appears to measure a recognisable aspect of the patients' quality of life. We will consider a question as contributing to a factor if it has a factor loading of at least 0.4 on that factor. Items not contributing to a factor in this way will be considered for rejection from the questionnaire.

\section{Validity}

Construct validity is a measure of the correlation of the scale being tested to another instrument that is believed to assess the same attributes with good validity and reliability. It is commonly measured using the Pearson correlation coefficient $(r)$, with a low correlation suggesting that the tests either have low validity or are measuring different things and a high correlation suggesting that the tests are very similar and a new scale is not needed. ${ }^{22}$ We will accept the construct validity of the FACE-Q Skin Cancer Module if the Pearson correlation is $0.4-0.8^{3}$ when tested against the Facial Skin Cancer Index ${ }^{17}$ and the generic EQ5D. ${ }^{18}$ If the anglicised FACE-Q and its subscales are valid measures to assess facial reconstruction we would expect to see small to moderate levels of correlation with the general health questionnaires. In addition, if any of the FACE-Q subscales are valid measures of facial reconstruction we might expect to see, for example, worse scores on aesthetic subscales for patients who have undergone a more extensive surgical operation than those who have had less invasive surgery.

\section{Reproducibility}

Reproducibility is another measure of test reliability; in this case, the reliability of the test to give similar scores on two or more occasions in the same person assuming 
their condition has not changed. We will assess reproducibility by comparing patients' FACE-Q Skin Cancer Module scores at two time points postsurgery when the patient has stabilised, to a subsample of around $20 \%$ of the study population. We will administer the questionnaire at two time points postsurgery, a period of 1-2 weeks apart. In addition to completing the FACE-Q Skin Cancer Module at the second time point, each patient will be asked whether they consider their quality of life to have improved, deteriorated or stayed the same. We would expect that for patients who report no change in their symptoms that the scores will be consistent at these two time points. We will assess the reproducibility of the scores for stable patients using intraclass correlation, with a value of 0.75 acceptable. ${ }^{19}$

\section{Responsiveness}

Responsiveness in the instrument is its ability to detect change in a patient's condition. We will assess responsiveness of the FACE-Q Skin Cancer Module in those patients' reporting either an improvement or deterioration in their condition pre-surgery and postsurgery. We will use Guyatt's responsiveness statistics to quantify the responsiveness of the FACE-Q Skin Cancer Module. ${ }^{23}$

In order to determine if the instrument is both valid and sensitive enough to compare different types of reconstruction, we will split the recruited patients into three population groups (those that undergo direct closure of the wound, skin grafting or local flap) and compare FACE-Q Skin Cancer Module total and subscale scores to determine if the questionnaire is sensitive enough to detect differences in scores between these three groups. We will use analysis of variance or non-parametric equivalent tests (depending on sample size) to determine if any differences exist.

Contributors TD, ISW and HAH: developed and designed the study. TD: produced the initial draft of this paper. All authors: involved in critically appraising and editing the article; read and approved the final manuscript.

Funding TD is supported by the Welsh Clinical Academic Training (WCAT) scheme. This study is a collaboration between Swansea University and Abertawe Bro Morgannwg University (ABMU) Health Board.

Competing interests None declared.

Patient consent Obtained.

Ethics approval This study has been approved by the West Midlands-Edgbaston Research Ethics Committee (Ref 16/WM/0445).

Provenance and peer review Not commissioned; externally peer reviewed.

Open Access This is an Open Access article distributed in accordance with the Creative Commons Attribution Non Commercial (CC BY-NC 4.0) license, which permits others to distribute, remix, adapt, build upon this work non-commercially, and license their derivative works on different terms, provided the original work is properly cited and the use is non-commercial. See: http://creativecommons.org/ licenses/by-nc/4.0/ (c) Article author(s) (or their employer(s) unless otherwise stated in the text of the article) 2017. All rights reserved. No commercial use is permitted unless otherwise expressly granted.

\section{REFERENCES}

1. Geller AC, Annas GD. Epidemiology of melanoma and nonmelanoma skin cancer. Seminars in Oncology Nursing. W B Saunders 2003;19:2-11.

2. Skincancer.org. (accessed 21 Jan 2017).

3. Cancer Research UK statistics. 2016:1. http://www. cancerresearchuk.org/health-professional/cancer-statistics/statisticsby-cancer-type/skin-cancer/incidence (accessed 21 Jan 2017).

4. Rogers HW, Weinstock MA, Feldman SR, et al. Incidence estimate of nonmelanoma skin cancer (Keratinocyte Carcinomas) in the US population, 2012. JAMA Dermatol 2015;151:1081-6.

5. Franceschi S, Levi F, Randimbison L, et al. Site distribution of different types of skin cancer: new aetiological clues. Int J Cancer 1996;67:24-8.

6. Santmyire BR, Feldman SR, Fleischer AB. Lifestyle high-risk behaviors and demographics may predict the level of participation in sun-protection behaviors and skin cancer primary prevention in the united states. Cancer 2001;92:1315-24.

7. Kolk A, Wolff KD, Smeets R, et al. Melanotic and non-melanotic malignancies of the face and external ear - a review of current treatment concepts and future options. Cancer Treat Rev 2014;40:819-37.

8. Haque T, Rahman KM, Thurston DE, et al. Topical therapies for skin cancer and actinic keratosis. Eur J Pharm Sci 2015;77:279-89.

9. Fort M, Guet S, Colson-Durand L, et al. Role of radiation therapy in non-melanoma cancers, lymphomas and sarcomas of the skin: systematic review and best practice in 2016. Crit Rev Oncol Hematol 2016;99:200-13.

10. Shokrollahi K, Javed M, Aeuyung K, et al. Combined Carbon Dioxide laser with photodynamic therapy for nodular and superficial basal cell carcinoma. Ann Plast Surg 2014;73:552-8.

11. Madan V, Lear JT, Szeimies R-M. Non-melanoma skin cancer. The Lancet 2010;375:673-85.

12. Lansley A. Equity and excellence: liberating the NHS: Department of Health, 2010.

13. Devlin N. Getting the most out of PROMs: health outcomes and NHS decision-making, Nancy Devlin and John Appleby. The King's Fund 2010;5:1-92.

14. Gibbons E, Casañas i Comabella C, Fitzpatrick R. A structured review of patient-reported outcome measures for patients with skin cancer, 2013. Br J Dermatol 2013;168:1176-86.

15. Lee EH, Klassen AF, Lawson JL, et al. Patient experiences and outcomes following facial skin cancer surgery: A qualitative study. Australas J Dermatol 2016;57:e100-4.

16. Wild D, Grove A, Martin M, et al. Principles of good practice for the translation and cultural adaptation process for Patient-Reported Outcomes (PRO) measures: report of the ISPOR task force for translation and cultural adaptation. Value Health 2005;8:94-104.

17. Matthews BA, Rhee JS, Neuburg M, et al. Development of the facial skin care index: a health-related outcomes index for skin cancer patients. Dermatol Surg 2006;32:924-34.

18. EuroQol Group. EuroQol-a new facility for the measurement of health-related quality of life. Health Policy 1990;16:199-208.

19. Streiner DL, Norman GR. Health measurement scales: a practical guide to their development and use 4 edition Oxford University Press. New York 2008.

20. Barrett PT, Kline P. The observation to variable ratio in factor analysis. Personality study and group behavior. 1981.

21. Aaronson N, Alonso J, Burnam A, et al. Assessing health status and quality-of-life instruments: attributes and review criteria. Qual Life Res 2002;11:193-205.

22. Marx RG, Bombardier C, Hogg-Johnson S, et al. Clinimetric and psychometric strategies for development of a health measurement scale. J Clin Epidemiol 1999;52:105-11.

23. Deyo RA, Diehr P, Patrick DL. Reproducibility and responsiveness of health status measures. Statistics and strategies for evaluation. Control Clin Trials 1991;12:S142-58. 